\title{
Toward Rational Design of Ionic Liquid/Metal-Organic Framework Composites: Effects of Interionic Interaction Energy
}

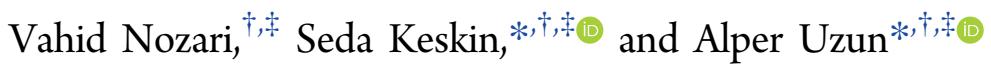 \\ ${ }^{\dagger}$ Department of Chemical and Biological Engineering and ${ }^{\ddagger}$ Koç University TÜPRAŞ Energy Center (KUTEM), Koç University, \\ Rumelifeneri Yolu, 34450 Sariyer, Istanbul, Turkey
}

\section{Supporting Information}

\begin{abstract}
One of the structural factors controlling the extent of interactions between ionic liquids (ILs) and metal-organic frameworks (MOFs) in IL/MOF composites is elucidated. Results showed that the thermal stability limits and adsorption performances of the IL/MOF composites can be tuned by the interionic interaction energy of bulk ILs, which can be probed spectroscopically via $\mathrm{C} 2 \mathrm{H}$ infrared stretching frequency.
\end{abstract}

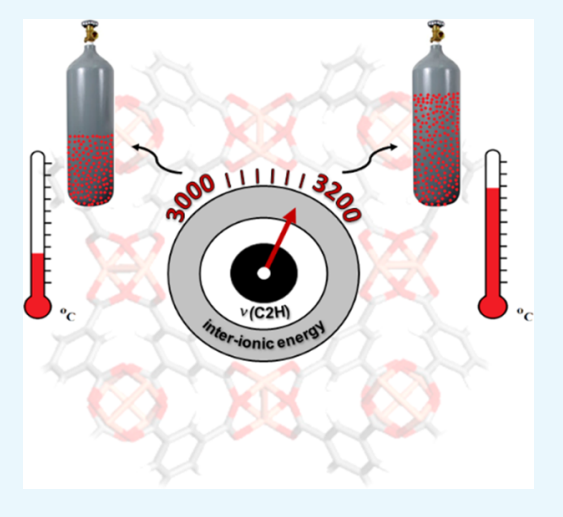

\section{INTRODUCTION}

Incorporation of ionic liquids (ILs) in metal-organic frameworks (MOFs) offers a broad potential in the way of improving the performance of the parent MOF material in various applications including catalysis, ${ }^{1,2}$ ionic conductivity, ${ }^{3}$ gas storage, ${ }^{4}$ and gas separation. ${ }^{5}$ Among these applications, the outcomes in gas storage and gas separation are especially promising. Here, the key point is that ILs have tunable physicochemical properties, presenting opportunities for adjusting the characteristics of the available adsorption sites in MOFs. ${ }^{6,7}$ Moreover, the presence of an IL molecule inside the MOF cages also offers possibilities of regulating the available pore space for the gas adsorption. Consequently, gas storage and gas separation performance of the parent MOF can be tuned by the proper choice of IL/MOF pairs. For instance, we recently incorporated 1-n-butyl-3-methylimidazolium tetrafluoroborate [BMIM] $\left[\mathrm{BF}_{4}\right]$ into CuBTC, HKUST-1 $\left(\mathrm{Cu}_{3}(\mathrm{BTC})_{2}\right.$; $\mathrm{BTC}=$ benzene-1,3,5-tricarboxylate) ${ }^{8}$ High-pressure volumetric uptake measurements illustrated that the incorporation of $[\mathrm{BMIM}]\left[\mathrm{BF}_{4}\right]$ increases $\mathrm{CH}_{4}$ selectivity over $\mathrm{CO}_{2}, \mathrm{H}_{2}$, and $\mathrm{N}_{2}$ by more than 1.5 times in comparison with their corresponding values in pristine CuBTC. ${ }^{6}$

Similarly, incorporation of another IL, 1-n-butyl-3-methylimidazolium hexafluorophosphate $[\mathrm{BMIM}]\left[\mathrm{PF}_{6}\right]$, into another MOF, zeolitic imidazolate framework (ZIF-8), led to a more significant increase in gas separation performance. ${ }^{7}$ Results showed that $\mathrm{CO}_{2} / \mathrm{CH}_{4}$ and $\mathrm{CO}_{2} / \mathrm{N}_{2}$ selectivities increased from 2.2 and 6.5 to 8.9 and 24 , respectively, upon IL incorporation. Detailed characterization of the structures complemented by the density functional theory (DFT) calculations illustrated that there are strong interactions between the IL molecules and the host MOF. These results combined with the molecular simulations of gas adsorption in the IL/MOF composite showed that IL-MOF interactions are responsible for the improved gas separation performance. Similarly, incorporation of $[\mathrm{BMIM}]\left[\mathrm{BF}_{4}\right]$ into ZIF-8 led to an increase in $\mathrm{CO}_{2} / \mathrm{CH}_{4}$ selectivity from 2.2 to 4 and to the doubling of $\mathrm{CO}_{2} / \mathrm{N}_{2}$ selectivity from 6.5 to 13.2 . $^{9}$

These changes in gas adsorption and gas separation performances upon IL incorporation are directly controlled by the interactions between IL and MOF., ${ }^{7,9}$ Dhumal et al. ${ }^{10}$ confined $[\mathrm{EMIM}]\left[\mathrm{EtSO}_{4}\right]$ into $\mathrm{CuBTC}$ and investigated the interactions by Fourier transform infrared (FTIR) spectroscopy complemented with DFT calculations. They showed that interactions have a significant effect on the structure of the MOF as well as on the IL. Accordingly, IL preferentially interacts with $\mathrm{Cu}$ ions, and this interaction weakens the $\mathrm{Cu}-\mathrm{O}$ bonding. Besides these studies, computational efforts have been investigating the IL/MOF composites. Vicent-Luna et al. ${ }^{11}$ focused on five different ILs with 1-ethyl-3-methylimidazolium $[\mathrm{EMIM}]^{+}$cation and performed molecular simulations to evaluate the effect of the IL incorporation in a MOF, CuBTC, on the $\mathrm{CO}_{2}$ separation performance. They illustrated an enhancement in the $\mathrm{CO}_{2}$ adsorption at lower pressures compared to pristine CuBTC. $\mathrm{Li}$ et al. ${ }^{5}$ also performed molecular simulations to study the IL/Cu-TDPAT composites for $\mathrm{H}_{2} \mathrm{~S} / \mathrm{CH}_{4}$ separation. The ILs consisted of an identical cation, 1- $n$-butyl-3-methylimidazolium $[\mathrm{BMIM}]^{+}$, and different anions $\left([\mathrm{Cl}]^{-},\left[\mathrm{Tf}_{2} \mathrm{~N}\right]^{-},\left[\mathrm{PF}_{6}\right]^{-}\right.$, and $\left.\left[\mathrm{BF}_{4}\right]^{-}\right)$. They showed

Received: July 27, 2017

Accepted: September 28, 2017

Published: October 11, 2017 
that incorporation of ILs could increase the $\mathrm{H}_{2} \mathrm{~S}$ adsorption compared to pristine MOF.

All of these studies illustrate that combining different ILs with different MOFs leads to composite materials with various gas adsorption and separation performances. The research on applications of IL/MOF composites is rapidly growing. ${ }^{12-15}$ One of the main challenges is that this field is too new and the rationale behind the proper choice of ILs and MOFs has not been fully understood yet. This fundamental information is crucial for the rational design of such materials. This is why there is a strong need for elucidating the structural factors controlling the interactions in $\mathrm{IL} / \mathrm{MOF}$ composites and their consequences on the gas storage and gas separation performance. In the way of contributing to overcome this challenge, here, we communicate a structural factor in ILs, which we identified to have a strong influence not only on the gas storage performances but also on the thermal stability limits of IL/ MOF composites.

When an IL is incorporated into the pores of MOFs, it interacts with the framework. This interaction can be physical and/or chemical based on the type of the IL and MOF. If the cation of the IL is fixed and the anion is changed, the cohesion energy between the cation and the anion can be systematically varied. As the cohesion energy between the ion moieties changes, the strength of hydrogen bond between the ions will vary. As a result of these changes in the strength of the hydrogen bond, the "interionic interaction energy" between the cation and the anion is modified. According to the previous studies in the literature, the strength of the interionic interaction energy between the cation and the anion can be probed by the stretching infrared (IR) frequency of the most acidic proton site on the imidazolium cation $(\mathrm{C} 2 \mathrm{H}$, where $\mathrm{C} 2$ is the carbon between two $\mathrm{N}$ sites in the ring). ${ }^{16-24}$ Motivated from this point, we examined the consequences of the changes in interionic interaction energy in bulk ILs on the thermal stability limits and gas adsorption performances of IL/MOF composites. For this purpose, we started with CuBTC, one of the most widely studied MOFs for IL incorporation as reviewed above. It has open metal sites that directly participate in gas adsorption process. Thus, we expected to observe a direct influence of the IL molecules on these sites when they are inside the pores. The ILs considered here were chosen from a family of ILs with the same cation, $[\mathrm{BMIM}]^{+}$, having different anions: bis(trifluoromethylsulfonyl)imide $\left[\mathrm{NTf}_{2}\right]^{-}$, methylsulfate $\left[\mathrm{MeSO}_{4}\right]^{-}$, hexafluoroantimonate $\left[\mathrm{SbF}_{6}\right]^{-}$, thiocyanate $[\mathrm{SCN}]^{-}$, trifluoromethanesulfonate $\left[\mathrm{CF}_{3} \mathrm{SO}_{3}\right]^{-}$, methanesulfonate $\left[\mathrm{MeSO}_{3}\right]^{-}$, and octylsulfate $\left[\mathrm{OcSO}_{4}\right]^{-}$. Because these ILs have the same cation, their interionic interaction strengths can be probed and quantified by the corresponding $\nu(\mathrm{C} 2 \mathrm{H})$ band position.

\section{RESULTS AND DISCUSSION}

Wet impregnation method was used for synthesizing the IL/ MOF composites with approximately $30 \mathrm{wt} \%$ stoichiometric IL loading. ${ }^{6,7,9}$ The resulting structures were fully characterized by X-ray fluorescence (XRF), X-ray diffraction (XRD), scanning electron microscopy (SEM), Fourier transform infrared (FTIR) spectroscopy, Brunauer-Emmett-Teller (BET) surface area analysis, Barrett-Joyner-Halenda $(\mathrm{BJH})$ pore size and volume analysis, and thermogravimetric analysis (TGA), as reported previously. ${ }^{6,7,9}$ From the BET measurements, we observed that the pore volume, and the BET surface area decreased in each IL/MOF sample as compared to pristine CuBTC (Table S1,
Supporting Information (SI)). This decrease indicates the presence of ILs inside the MOF, consistent with our previous reports. ${ }^{6,7,9}$ However, we note that the BET measurements are based on the $\mathrm{N}_{2}$ adsorption, which can be directly influenced by the presence of IL inside the MOF cages. Because $\mathrm{N}_{2}$ might be dissolved in different ILs at different levels, these BET results might be misleading in terms of providing the quantitative results. For instance, the IL loading calculations based on the change in the pore volumes upon IL incorporation fail to estimate the loadings. For instance, the estimated values for some of the samples were even higher than the stoichiometric amounts of individual components used in the synthesis (Table S1, SI). Thus, the IL loadings in each sample were determined by XRF, as listed in Table S1, SI. The XRD results and the SEM analysis, given in Figures S1 and S2, SI, confirmed that the crystal structure and the morphology of the CuBTC were preserved upon IL incorporation. Consistent with these results, the FTIR measurements (Figures S3-S9) also indicate that the IL and MOF structure remain intact with slight changes in the positions and intensities of some of the IR features. These changes were inferred to be related to the interactions between the IL molecules and the CuBTC. ${ }^{6,7,9}$ Because the IL is expected to interact directly with the unsaturated $\mathrm{Cu}$ sites, ${ }^{25,26}$ we focused on a band at $480 \mathrm{~cm}^{-1}$ associated with $(\mathrm{Cu}-\mathrm{O})$ stretching frequency, $\nu(\mathrm{Cu}-\mathrm{O})$. This band provides information regarding the electronic environment over the $\mathrm{Cu}$ sites, which should be influenced strongly by the presence of interactions between the IL and MOF. Thus, the $\nu(\mathrm{Cu}-\mathrm{O})$ band position was used as a probe to quantify these interactions. For instance, when the IL was [BMIM] [SCN], this band presented a red shift of approximately $9 \mathrm{~cm}^{-1}$. This red shift indicated a weakening in the $\mathrm{Cu}-\mathrm{O}$ bond probably because of the electron sharing between the unsaturated $\mathrm{Cu}$ atoms and the IL, leading to a decrease in the electron density on the $\mathrm{Cu}$ sites to be shared with the framework oxygen atoms. This result is consistent with those of Dhumal et al., ${ }^{10}$ who showed a decrease in the $\mathrm{Cu}-\mathrm{O}$ bond strength as a result of interactions between $\mathrm{IL}$ and $\mathrm{CuBTC}$.

As stated above, the ILs selected for this study have the same cation, $[\mathrm{BMIM}]^{+}$. The proton attached to the $\mathrm{C} 2$ position between the two nitrogen atoms of the imidazolium ring in $[\mathrm{BMIM}]^{+}$is the most acidic proton, which is responsible for strong directional and localized hydrogen bonding between the cation and anion of the IL. Therefore, its stretching frequency, $\nu(\mathrm{C} 2 \mathrm{H})$, can be used as a measure of interionic interaction energy in ILs. ${ }^{16-24}$ Accordingly, the interionic interaction energy becomes stronger with a red shift in $\nu(\mathrm{C} 2 \mathrm{H}){ }^{16,17,22,24}$ Thus, we evaluated the variations in $\nu(\mathrm{Cu}-\mathrm{O})$ in $\mathrm{IL} / \mathrm{MOF}$ composites with $\nu(\mathrm{C} 2 \mathrm{H})$ band position of the corresponding IL to elucidate the dependency of the electronic and bonding environment of the open metal sites in $\mathrm{CuBTC}$ on the interionic interaction energy in ILs.

Figure 1 illustrates that there is a correlation $\left(R^{2}=0.73\right)$ between the amount of red shift observed on $\nu(\mathrm{Cu}-\mathrm{O})$ band position and $\nu(\mathrm{C} 2 \mathrm{H})$ of ILs. Accordingly, the magnitude of red shift on $\nu(\mathrm{Cu}-\mathrm{O})$ increases as the interionic interaction energy in ILs increases (with a decrease in $\nu(\mathrm{C} 2 \mathrm{H})$ ). Thus, we infer that the $\mathrm{Cu}-\mathrm{O}$ bonding in the IL/MOF samples becomes weaker in the presence of IL. On the basis of the results presented in Figure 1, we infer that the degree of this weakening in $\mathrm{Cu}-\mathrm{O}$ bonding is the highest when the interionic interaction is the strongest. This behavior originates from the fact that the increase in interionic interactions in ILs is 


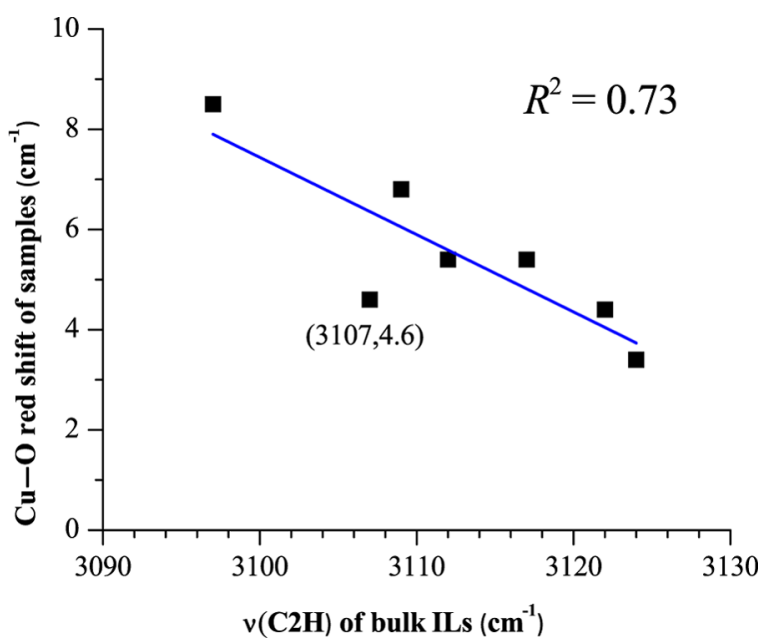

Figure 1. Correlation between $\nu(\mathrm{C} 2 \mathrm{H})$ of bulk ILs and $\mathrm{Cu}-\mathrm{O}$ red shifts in IL/MOF samples.

associated with an increase in the ionic character of the ions. This stronger ionic character enables ILs to interact more strongly with the unsaturated $\mathrm{Cu}$ sites, leading to a weakening in $\mathrm{Cu}-\mathrm{O}$ bonding (as evidenced by a red shift in $\nu(\mathrm{Cu}-\mathrm{O})$ ). Thus, we infer that the interionic interaction energy in ILs is an important structural parameter controlling the electronic environment over the gas adsorption sites in CuBTC. We note that among all the ILs considered here, only [BMIM]$\left[\mathrm{OcSO}_{4}\right]$ does not represent a good fit in Figure 1 (with a red shift in $\nu(\mathrm{Cu}-\mathrm{O})$ and $\nu(\mathrm{C} 2 \mathrm{H})$ values of $4.6 \mathrm{~cm}^{-1}$ and 3107 $\mathrm{cm}^{-1}$, respectively). This IL has a significantly large anion compared to others we considered in this work. ${ }^{27}$ Thus, the size of the anion should be another structural parameter that plays a role in sterically constraining the interactions between the IL and the open metal sites of the MOF. When excluding this specific IL from the fit presented in Figure 1, the correlation coefficient increases from 0.73 to 0.95 , presenting a very strong correlation.

These results suggest that one can tune the electronic environment over the open metal sites by changing the IL structure. As illustrated previously, ${ }^{7,28}$ it is also possible to adjust the available pore size and shape of the MOFs by the type and loading of IL. This ability offers a broad potential to control the performance of IL/MOF composites for various applications, such as gas adsorption, ${ }^{4}$ membrane-based gas separation, ${ }^{29}$ catalysis, ${ }^{1,2}$ and ionic conductivity. ${ }^{3}$ Because for these applications, the open metal sites are the sites for adsorption, their electronic environment directly determines the performance in the related application. In any of these applications, one of the key points is that the structure of the composite should remain intact under application conditions. Thus, we investigated the consequences of changes in interionic interaction energy in ILs on the thermal stability limits of these composites. Figure 2 illustrates the variation in $T^{\prime}{ }_{\text {onset }}$ of IL/ MOF composites, indicating the temperature at which the thermal decomposition starts, with $\nu(\mathrm{C} 2 \mathrm{H})$ band position of the corresponding IL.

Accordingly, the thermal stability limits of IL/MOF composites decrease linearly with an increase in the interionic interaction energy (probed by a decrease in $\nu(\mathrm{C} 2 \mathrm{H})$ ) associated with an increase in the degree of red shift in $\nu(\mathrm{Cu}-\mathrm{O})$. This strong correlation indicates that the interactions between the $\mathrm{IL}$ and open $\mathrm{Cu}$ sites are the

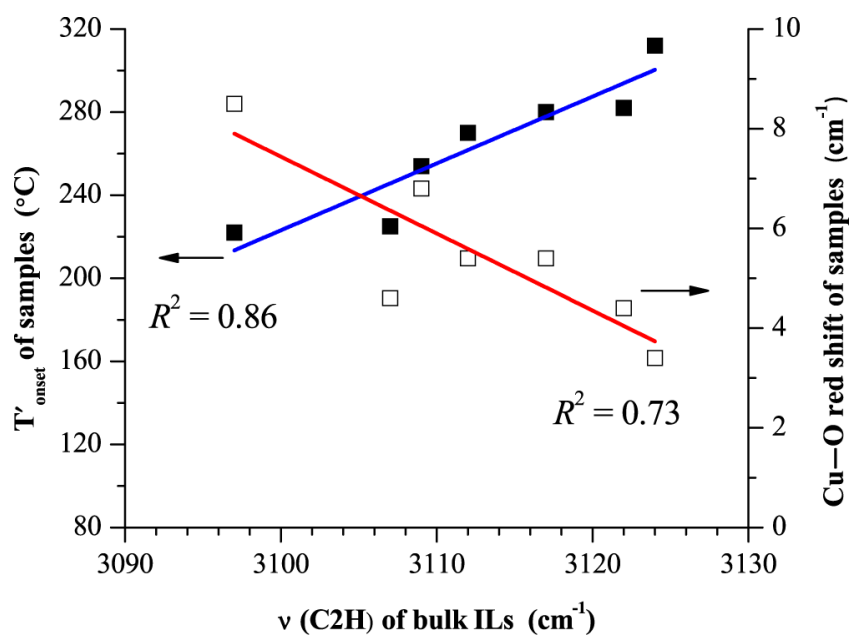

Figure 2. Correlation between $T^{\prime}$ onset of IL/MOF composites (left $y$ axis) and $\nu(\mathrm{C} 2 \mathrm{H})$ of bulk ILs. The vertical axis on the right shows the correlation between the corresponding red shift in $\nu(\mathrm{Cu}-\mathrm{O})$ and $\nu(\mathrm{C} 2 \mathrm{H})$ of bulk ILs.

dominant factors in determining the decomposition mechanism of the composite. Moreover, the correlation presented in Figure 2 can perfectly predict the decomposition temperature of an analogous [BMIM] $\left[\mathrm{BF}_{4}\right] / \mathrm{CuBTC}$ composite (with a corresponding $\nu(\mathrm{C} 2 \mathrm{H})$ value of $3124 \mathrm{~cm}^{-1}$ for $\left.[\mathrm{BMIM}]\left[\mathrm{BF}_{4}\right]^{9}\right)$ within a few degrees, confirming the validity of this correlation. ${ }^{6}$ Thus, we infer that the interionic interaction energy of the ILs has a strong influence on the characteristics of the $\mathrm{IL} / \mathrm{MOF}$ composites. When it is higher, the IL interacts with the $\mathrm{Cu}$ sites more strongly. This change lowers the stability limit, suggesting that interactions between IL-MOF play a major role in thermal decomposition.

Aiming at elucidating the corresponding influence on gas storage performance of the composites, we performed highpressure volumetric gas uptake measurements for $\mathrm{CO}_{2}$ and $\mathrm{CH}_{4}$. Here, we note that the gas uptake values were normalized per gram of CuBTC because our ultimate aim is to show how the presence of ILs influences the gas uptake performance of the MOF. In other words, IL-incorporated MOFs can be considered as functionalized MOFs and we aimed to directly compare the amount of gas being adsorbed by the functionalized material containing the same amount of MOF. Figure 3a illustrates the variation in $\mathrm{CO}_{2}$ uptake in $\mathrm{IL} / \mathrm{CuBTC}$ samples with an increase in $\nu(\mathrm{C} 2 \mathrm{H})$ of the IL at 1 bar. Data show that the $\mathrm{CO}_{2}$ uptake decreases with an increase in interionic interaction energy in ILs (with a decrease in $\nu(\mathrm{C} 2 \mathrm{H})$ ). As the open metal sites in CuBTC interact more strongly with the anion of the IL, they become less available for gas adsorption (as evidenced from a weakening of the $\mathrm{Cu}-\mathrm{O}$ bond). Confirming this inference, Figure $3 \mathrm{~b}$ illustrates a similar trend for $\mathrm{CH}_{4}$ uptake with even a higher degree of correlation. This result also suggests that these correlations strongly depend on the pressure.

Figure $3 c$ shows the $R^{2}$ values of these correlations as a function of pressure. Accordingly, the $R^{2}$ value for $\mathrm{CH}_{4}$ is as low as 0.63 at very low pressure region because of the weak interactions of $\mathrm{CH}_{4}$ molecules with the open metal sites at very low pressures. However, it quickly increases to values higher than 0.9 with increasing pressure. $\mathrm{CO}_{2}$, on the other hand, has quadrupole moments facilitating efficient interactions with the MOF at the very low pressure region. ${ }^{30}$ Thus, its $R^{2}$ starts from 

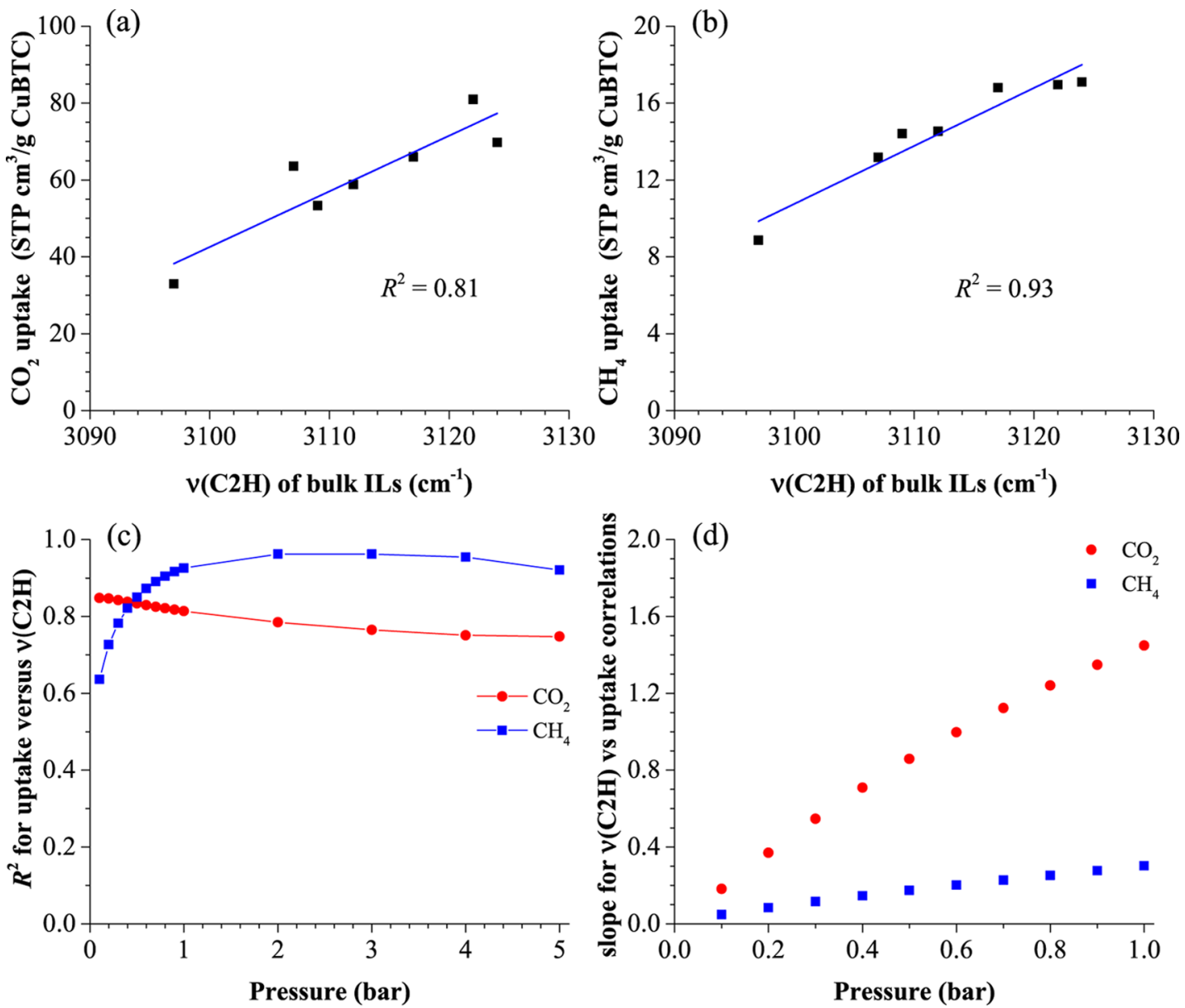

Figure 3. Correlation between $\mathrm{CH}_{4}$ and $\mathrm{CO}_{2}$ uptakes vs $\nu(\mathrm{C} 2 \mathrm{H})$ of bulk ILs: (a) $\mathrm{CO}_{2}$ uptake at 1 bar; (b) $\mathrm{CH}_{4}$ uptake at 1 bar; (c) correlation coefficients for $\mathrm{CO}_{2}$ uptake and $\mathrm{CH}_{4}$ uptake at different pressures (a line was added between the points to guide the eye); (d) slope for correlations between $\nu(\mathrm{C} 2 \mathrm{H})$ and gas uptakes.

a value above 0.8 at very low pressures and gradually decreases with increasing pressure. We note that at high pressures, the available pore space is the dominant factor for gas adsorption, as evidenced by a decrease in $R^{2}$ with increasing pressure. Moreover, Figure $3 \mathrm{~d}$ represents the change in slope of correlations between the corresponding gas uptakes in IL/ MOF composites and $\nu(\mathrm{C} 2 \mathrm{H})$ of the ILs. Data show that the slope for the correlation of $\mathrm{CO}_{2}$ uptake with $\nu(\mathrm{C} 2 \mathrm{H})$ is always higher than that of $\mathrm{CH}_{4}$ uptake. Thus, we infer that the interionic interaction energy in ILs plays a dominant role in controlling the $\mathrm{CO}_{2}$ uptake; however, it is not that effective for controlling $\mathrm{CH}_{4}$ uptake, especially at low pressures. Similar to the case with the decomposition temperature, $\mathrm{CH}_{4}$ and $\mathrm{CO}_{2}$ uptakes for a previously reported analogous [BMIM] $\left[\mathrm{BF}_{4}\right] /$ CuBTC composite $^{6}$ can be estimated within less than $\pm 5 \%$ error from the correlations presented in Figure 3, confirming their validity.

\section{CONCLUSIONS}

Results presented here illustrate that the interionic interaction energy in ILs is a major contributor in determining the interactions of ILs with MOFs. These interactions directly control the electronic environment over the unsaturated metal sites, which act as the gas adsorption sites in CuBTC. Results also illustrated that these interactions play a major role in determining the thermal stability limits and the gas storage performance. The fundamental understanding presented here can be extended to other IL and MOF combinations to fully elucidate the structure-interactions-performance relationships in IL/MOF composites. This information will be a guide in the rational design of these composite materials with exceptional performance for any desired application. Existence of many possible IL structures provides tunable physical and chemical properties. Combining them with a large number of different MOFs with various chemistry and topology offers a broad potential.

\section{EXPERIMENTAL SECTION}

4.1. Materials and Sample Preparation. CuBTC (Basolite C300), ILs, and acetone were purchased from Sigma-Aldrich. All of the materials were stored in an Ar-filled glovebox (Labconco). ILs were dried at $353 \mathrm{~K}$ for $6 \mathrm{~h}$ and kept under vacuum for $12 \mathrm{~h}$ before transferring it in to the glovebox. At first, CuBTC was dried overnight in a vacuum oven at 105 ${ }^{\circ} \mathrm{C}$. IL-incorporated samples were prepared using acetone as solvent. Each IL (300 mg) was first dissolved in $20 \mathrm{~mL}$ acetone and stirred at room temperature for $1 \mathrm{~h}$, followed by the addition of $700 \mathrm{mg}$ freshly dried CuBTC. The resulting samples were stirred for $6 \mathrm{~h}$ at $35{ }^{\circ} \mathrm{C}$ until acetone had almost evaporated. The samples were dried in an oven at $105{ }^{\circ} \mathrm{C}$ overnight. ${ }^{6}$ 
4.2. Fourier Transform Infrared (FTIR) Spectroscopy. A Thermo Scientific Nicolet iS50 model FTIR spectrometer with an attenuated total reflection cell was used to collect the IR spectra of bulk ILs and incorporated samples. Five hundred and twelve scans with $2 \mathrm{~cm}^{-1}$ resolution were collected and averaged at room temperature. Before each measurement, 128 background scans were collected and averaged. Peak analyses and deconvolutions were performed using Fityk software. ${ }^{31}$

4.3. X-ray Diffraction. A Bruker D8 Phaser instrument with an X-ray generator performance of $30 \mathrm{kV}$ of voltage and $10 \mathrm{~mA}$ of current was utilized to obtain the diffraction pattern of samples. A $\mathrm{Cu} \mathrm{K} \alpha 1$ radiation source with a wavelength of $1.5418 \AA$ was used and a Lynxeye detector was used with a slit size of $1 \mathrm{~mm}$. Data were collected for each sample between 5 and $49^{\circ}$, with a step size of $0.0204^{\circ}$.

4.4. Brunauer-Emmett-Teller (BET). A Micromeritics ASAP 2020 physisorption analyzer was used for the BET analysis. Approximately $150 \mathrm{mg}$ of IL/MOF composites were used for the measurements. Samples were activated at $125{ }^{\circ} \mathrm{C}$ under vacuum. Subsequently, the samples were cooled down to liquid nitrogen temperature and then the free space measurement was performed using $\mathrm{He} . \mathrm{N}_{2}$ gas adsorption isotherms were obtained by feeding the gas in the range of $10^{-6}$ to 1 bar. The pressure steps between 0.08 and 0.25 bar were used to fit the BET equation to estimate the surface areas of the samples. Pore volumes of the composite materials were obtained from the $\mathrm{N}_{2}$ adsorption isotherm at $77 \mathrm{~K}$ using the t-plot method.

4.5. Thermogravimetric Analysis (TGA). A TA Instruments Q500 model thermogravimetric analyzer was used with a platinum pan. After taring the empty clean pan, approximately $15-20 \mathrm{mg}$ of samples including pure ILs and incorporated samples were weighted. A temperature ramp rate of $5{ }^{\circ} \mathrm{C} / \mathrm{min}$ was employed up to $120{ }^{\circ} \mathrm{C}$ following an isotherm treatment at $120^{\circ} \mathrm{C}$ for $8 \mathrm{~h}$ and then the temperature was ramped to $700{ }^{\circ} \mathrm{C}$ at a rate of $2{ }^{\circ} \mathrm{C} / \mathrm{min}$ under $\mathrm{N}_{2}$ flow rates of 40 and $60 \mathrm{~mL} / \mathrm{min}$ for balance and purge gases, respectively. For the comparison of thermal decomposition temperatures, the onset $\left(T_{\text {onset }}\right)$ and derivative onset $\left(T^{\prime}{ }_{\text {onset }}\right)$ temperatures were determined by extrapolation from thermogravimetry (TG) and derivative TG curves. $T_{\text {onset }}$ generally overestimates the thermal decomposition temperature compared to $T^{\prime}{ }_{\text {onset }}$ values. Consequently, we have considered $T^{\prime}$ onset values in this study.

4.6. Scanning Electron Microscope. A Zeiss Evo LS 15 scanning electron microscope was used for imaging the morphology of pure CuBTC and IL-incorporated samples. During the analysis, a voltage of $3 \mathrm{kV}$ was applied under vacuum. Working distance was approximately $6 \mathrm{~mm}$ for both CuBTC and IL/CuBTC composites. We prepared the samples in a way that about $5 \mathrm{mg}$ of each sample was placed on the carbon tape pasted on a cell.

4.7. X-ray Fluorescence (XRF). A Bruker S8 Tiger XRF spectrometer was used for elemental analysis of IL/MOF samples. ${ }^{7}$ For each of the IL/MOF composites, a container (Chemplex Industries Inc.) was filled with approximately 150 mg of powder sample and a $4 \mu \mathrm{m}$ Prolene film support (Chemplex Industries Inc.) was placed on top of the container and a ring was used to immobilize the film. Under helium atmosphere, X-rays were generated by using a $4 \mathrm{~kW}$ Rh anode $\mathrm{X}$-ray tube. Elemental compositions were determined using an $18 \mathrm{~mm}$ mask. Common ppm level impurities for almost all of the samples were Os, La, Rh, and Ca. The SpectraPlus Eval2 V2.2.454 software was used for data interpretation. CHO was chosen as the matrix for all of the composites. According to the weight percentage of each element in the composite, an element that was presented only in the IL structure was used to obtain the corresponding IL amount, and CuBTC amount was calculated from the weight percentage of the $\mathrm{Cu}$ atom.

4.8. Gas Adsorption Measurements. We used a Micromeritics (Particulate Systems) High Pressure Volumetric Analyzer HPVA II- $200^{32}$ for measuring the gas adsorption performance of the IL/MOF composites. Approximately $0.35 \mathrm{~g}$ sample was weighted and degassed at $125{ }^{\circ} \mathrm{C}$ under vacuum until the pressure reaches $10^{-6}$ bar. The stabilities of ILs after degassing the samples at this temperature were confirmed according to IR and TGA measurements. ${ }^{7}$ After degassing, the sample holder was connected to the analysis port and the temperature was set to $25{ }^{\circ} \mathrm{C}$ prior to measurements; all of the lines were purged with $\mathrm{He}$ three times to clean and remove any residuals from the previous experiments. The adsorption measurements were performed for a pressure range of $0.1-$ 30 bar. The obtained isotherms were fitted to Freundlich model for each sample.

\section{ASSOCIATED CONTENT}

\section{Supporting Information}

The Supporting Information is available free of charge on the ACS Publications website at DOI: 10.1021/acsomega.7b01074.

Additional data on characterization (Figures S1-S16 and Tables S1 and S2) (PDF)

\section{AUTHOR INFORMATION}

\section{Corresponding Authors}

*E-mails: skeskin@ku.edu.tr (S.K.).

*E-mails: auzun@ku.edu.tr (A.U.).

ORCID

Seda Keskin: 0000-0001-5968-0336

Alper Uzun: 0000-0001-7024-2900

Notes

The authors declare no competing financial interest.

\section{ACKNOWLEDGMENTS}

This work is supported by the Scientific and Technological Research Council of Turkey (TUBITAK) under 1001-Scientific and Technological Research Projects Funding Program (project number: 114R093) and by Koç University Seed Fund Program. Support provided by the KUTEM (Koç University TÜPRAS Energy Center) is gratefully acknowledged. A.U. acknowledges the TUBA-GEBIP Award. Supports of Çiğdem Altıntaş, Burak Koyutürk, and Fatma Pelin Kınık are gratefully acknowledged. Authors thank Koç University Surface Science and Technology Center (KUYTAM) for providing help with the sample characterization. Authors would like to thank TARLA for the collaborative research support.

\section{REFERENCES}

(1) Luo, Q.-x.; Ji, M.; Lu, M. H.; Hao, C.; Qiu, J. S.; Li, Y. Q. Organic Electron-Rich N-Heterocyclic Compound as a Chemical Bridge: Building a Bronsted Acidic Ionic Liquid Confined in MIL-101 Nanocages. J. Mater. Chem. A 2013, 1, 6530-6534.

(2) Han, M.; Gu, Z.; Chen, C.; Wu, Z.; Que, Y.; Wang, Q.; Wan, H.; Guan, G. Efficient Confinement of Ionic Liquids in MIL-100(Fe) Frameworks by the "Impregnation-Reaction-Encapsulation" Strategy for Biodiesel Production. RSC Adv. 2016, 6, 37110-37117.

(3) Chen, L.-H.; Wu, B.-B.; Zhao, H.-X.; Long, L.-S.; Zheng, L.-S. High Temperature Ionic Conduction Mediated by Ionic Liquid 
Incorporated within the Metal-Organic Framework UiO-67(Zr). Inorg. Chem. Commun. 2017, 81, 1-4.

(4) da Silva, F. W. M.; Magalhães, G. M.; Jardim, E. O.; SilvestreAlbero, J.; Sepúlveda-Escribano, A.; de Azevedo, D. C. S.; de Lucena, S. M. P. $\mathrm{CO}_{2}$ Adsorption on Ionic Liquid - Modified Cu-BTC: Experimental and Simulation Study. Adsorpt. Sci. Technol. 2015, 33, 223-242.

(5) Li, Z.; Xiao, Y.; Xue, W.; Yang, Q.; Zhong, C. Ionic Liquid/MetalOrganic Framework Composites for $\mathrm{H}_{2} \mathrm{~S}$ Removal from Natural Gas: A Computational Exploration. J. Phys. Chem. C 2015, 119, 3674-3683.

(6) Sezginel, K. B.; Keskin, S.; Uzun, A. Tuning the Gas Separation Performance of CuBTC by Ionic Liquid Incorporation. Langmuir 2016, 32, 1139-1147.

(7) Kinik, F. P.; Altintas, C.; Balci, V.; Koyuturk, B.; Uzun, A.; Keskin, S. [BMIM] $\left[\mathrm{PF}_{6}\right]$ Incorporation Doubles $\mathrm{CO}_{2}$ Selectivity of ZIF-8: Elucidation of Interactions and Their Consequences on Performance. ACS Appl. Mater. Interfaces 2016, 8, 30992-31005.

(8) Chui, S. S.-Y.; Lo, S. M.-F.; Charmant, J. P. H.; Orpen, A. G.; Williams, I. D. A Chemically Functionalizable Nanoporous Material $\left[\mathrm{Cu}_{3}(\mathrm{TMA})_{2}\left(\mathrm{H}_{2} \mathrm{O}\right)_{3}\right]$ n. Science 1999, 283, 1148-1150.

(9) Koyuturk, B.; Altintas, C.; Kinik, F. P.; Keskin, S.; Uzun, A. Improving Gas Separation Performance of ZIF-8 by $[\mathrm{BMIM}]\left[\mathrm{BF}_{4}\right]$ Incorporation: Interactions and Their Consequences on Performance. J. Phys. Chem. C 2017, 121, 10370-10381.

(10) Dhumal, N. R.; Singh, M. P.; Anderson, J. A.; Kiefer, J.; Kim, H. J. Molecular Interactions of a Cu-Based Metal Organic Framework with a Confined Imidazolium-Based Ionic Liquid: A Combined Density-Functional Theory and Experimental Vibrational Spectroscopy Study. J. Phys. Chem. C 2016, 120, 3295-3304.

(11) Vicent-Luna, J. M.; Gutiérrez-Sevillano, J. J.; Anta, J. A.; Calero, S. Effect of Room-Temperature Ionic Liquids on $\mathrm{CO}_{2}$ Separation by a Cu-BTC Metal-Organic Framework. J. Phys. Chem. C 2013, 117, 20762-20768.

(12) Ma, J.; Ying, Y.; Guo, X.; Huang, H.; Liu, D.; Zhong, C. Fabrication of Mixed-Matrix Membrane Containing Selective and Facilitated $\mathrm{CO}_{2}$ Transport Metal-Organic Framework Composite with Task-Specific Ionic Liquid for Efficient $\mathrm{CO}_{2}$ Separation. J. Mater. Chem. A 2016, 4, 7281-7288.

(13) Xue, W.; Li, Z.; Huang, H.; Yang, Q.; Liu, D.; Xu, Q.; Zhong, C. Effects of Ionic Liquid Dispersion in Metal-Organic Frameworks and Covalent Organic Frameworks on $\mathrm{CO}_{2}$ Capture: A Computational Study. Chem. Eng. Sci. 2016, 140, 1-9.

(14) Chen, Y.; Hu, Z.; Gupta, K. M.; Jiang, J. Ionic Liquid/MetalOrganic Framework Composite for $\mathrm{CO}_{2}$ Capture: A Computational Investigation. J. Phys. Chem. C 2011, 115, 21736-21742.

(15) Khan, N. A.; Hasan, Z.; Jhung, S. H. Ionic Liquids Supported on Metal-Organic Frameworks: Remarkable Adsorbents for Adsorptive Desulfurization. Chem. - Eur. J. 2014, 20, 376-380.

(16) Babucci, M.; Uzun, A. Effects of Interionic Interactions in 1,3Dialkylimidazolium Ionic Liquids on the Electronic Structure of Metal Sites in Solid Catalysts with Ionic Liquid Layer (SCILL). J. Mol. Liq. 2016, 216, 293-297.

(17) Babucci, M.; Fang, C.-Y.; Hoffman, A. S.; Bare, S. R.; Gates, B. C.; Uzun, A. Tuning the Selectivity of Single-Site Supported Metal Catalysts with Ionic Liquids. ACS Catal. 2017, 6969-6972.

(18) Babucci, M.; Akçay, A.; Balci, V.; Uzun, A. Thermal Stability Limits of Imidazolium Ionic Liquids Immobilized on Metal-Oxides. Langmuir 2015, 31, 9163-9176.

(19) Akçay, A.; Babucci, M.; Balci, V.; Uzun, A. A Model to Predict Maximum Tolerable Temperatures of Metal-Oxide-Supported 1-NButyl-3-Methylimidazolium Based Ionic Liquids. Chem. Eng. Sci. 2015, 123, 588-595.

(20) Akçay, A.; Balci, V.; Uzun, A. Structural Factors Controlling Thermal Stability of Imidazolium Ionic Liquids with 1-N-Butyl-3Methylimidazolium Cation on $\gamma-\mathrm{Al}_{2} \mathrm{O}_{3}$. Thermochim. Acta 2014, 589, 131-136.

(21) Noack, K.; Schulz, P. S.; Paape, N.; Kiefer, J.; Wasserscheid, P.; Leipertz, A. The Role of the C2 Position in Interionic Interactions of
Imidazolium Based Ionic Liquids: A Vibrational and NMR Spectroscopic Study. Phys. Chem. Chem. Phys. 2010, 14153-14161.

(22) Fumino, K.; Wulf, A.; Ludwig, R. The Cation-Anion Interaction in Ionic Liquids Probed by Far-Infrared Spectroscopy. Angew. Chem., Int. Ed. 2008, 47, 3830-3834.

(23) Fumino, K.; Wulf, A.; Ludwig, R. Strong, Localized, and Directional Hydrogen Bonds Fluidize Ionic Liquids. Angew. Chem., Int. Ed. 2008, 47, 8731-8734.

(24) Wulf, A.; Fumino, K.; Ludwig, R. Spectroscopic Evidence for an Enhanced Anion-Cation Interaction from Hydrogen Bonding in Pure Imidazolium Ionic Liquids. Angew. Chem., Int. Ed. 2010, 49, 449-453.

(25) Karra, J. R.; Walton, K. S. Effect of Open Metal Sites on Adsorption of Polar and Nonpolar Molecules in Metal-Organic Framework Cu-BTC. Langmuir 2008, 24, 8620-8626.

(26) Dietzel, P. D. C.; Besikiotis, V.; Blom, R. Application of MetalOrganic Frameworks with Coordinatively Unsaturated Metal Sites in Storage and Separation of Methane and Carbon Dioxide. J. Mater. Chem. 2009, 19, 7362.

(27) Materials Studio, v8.0. Materials Studio Manual; Biovia Software Inc., 2007.

(28) Ban, Y.; Li, Z.; Li, Y.; Peng, Y.; Jin, H.; Jiao, W.; Guo, A.; Wang, P.; Yang, Q.; Zhong, C.; et al. Confinement of Ionic Liquids in Nanocages: Tailoring the Molecular Sieving Properties of ZIF-8 for Membrane-Based $\mathrm{CO}_{2}$ Capture. Angew. Chem., Int. Ed. 2015, 54, 15483-15487.

(29) Gupta, K. M.; Chen, Y.; Hu, Z.; Jiang, J. Metal-Organic Framework Supported Ionic Liquid Membranes for $\mathrm{CO}_{2}$ Capture: Anion Effects. Phys. Chem. Chem. Phys. 2012, 14, 5785-5794.

(30) Uzun, A.; Keskin, S. Site Characteristics in Metal Organic Frameworks for Gas Adsorption. Prog. Surf. Sci. 2014, 89, 56-79.

(31) Wojdyr, M. Fityk: A General-Purpose Peak Fitting Program. J. Appl. Crystallogr. 2010, 43, 1126-1128.

(32) HPVA-II-High-Pressure-Volumetric-Analyzer. HPVA-II-HighPressure-Volumetric-Analyzer @ Particulate systems.com, 2016. 